\title{
The galvanic skin response orienting reflex among chronic schizophrenics
}

\author{
Alvin S. Bernstein I \\ FRANKLIN DELANO ROOSEVELT V. A. HOSPITAL, MONTROSE, N. Y.
}

\begin{abstract}
Abstraet
Regressed schizophrenics showed marked reduction in both OR frequency and amplitude in relation to both controls and remitted schizophrenics. However, initiai response frequency increased virtually to control levels when a more intense stimulus was used. All schizophrenics in common habituated faster than controls. Increased hospitalization was itself associated with reduced overall OR reactivity though phenothiazine medication had no significant effect. All results were supported on re-test some two months later.
\end{abstract}

\section{Problem}

The orienting reflex (OR), triggered by change or novelty in any stimulus dimension within the perceptual field, is believed to play a basic role in maintaining the organism in stable relations with his changing environment. Specifically, Sokolov (1963), suggests that the trigger mechanism is a "mis-match" between sensory input and a "neuronal model" of the perceptual field. The prime function of this reflex lies in its speed-up of the absorption and transmission of information within the nervous system, heightening the organism's capacity to cope with the recurrent "strangenesses" encountered in a fluid environment. OR activity habituates as the model is altered to incorporate novel stimulus aspects, re-appearing when mis-match is again encountered (Sokolov et al, 1961).

Since schizophrenia prominently involves impairment in response to the external world, some defect in OR function might be expected. In fact, such impairment has been reported by the Russians (Lynn, 1963). This paper compares OR arousal and habituation among chronic hospitalized schizophrenics and healthy controls. The influences of schizophrenic regression, phenothiazine treatment, and varying stimulus intensity were also examined.

\footnotetext{
Method

The GSR OR component was measured in response to photic stimuli. Five samples were used: 48 healthy controls, 60 remitted, largely symptom-free, and 60 regressed, highly disorganized schizophrenics, with the latter two subdivided into drug (currently receiving one of the phenothiazines), and nondrug (drug free at least 30 days before examination), samples. Mean ages were: normal, 38.5 years; remitted patients, 41.6; regressed patients, 41.2; drug patients, 40.3; nondrug patients, 42.4. Mean lengths of hospitalization were: remitted patients, 8.9 years; regressed patients, 14.4; drug patients, 11.3; nondrug, 12.1. Only the difference in hospitalization between remitted and regressed patients is significant $(p<.01)$. The remittedregressed distinction was based on extreme ratings by both ward physician and psychologist. All Ss were free of CNS damage.

Ss sat facing a rear-projection screen in a semi-darkened, sounddeadened room. An assistant signalled whenever $\mathrm{S}$ was not addressing the screen with eyes open. Stainless-steel finger electrodes were
}

applied with Cambridge paste. Measurement was through an Airborne Instruments Resistance Monitor with signals recorded on a Texas Instruments Recti/Riter.

After a 5-min. adaptation period, a tachistoscope presented a 1-sec. flash of light, repeated 10 times at irregular intervals from 15 to $60 \mathrm{sec}$. Half of each sample received $5 \mathrm{ft}$-candle intensity, half, a $25 \mathrm{ft}$-candle intensity. OR, scored as change in log conductance, was defined as a deflection of at least 500 ohms occurring 1-3 sec. after stimulus onset.

Results

All differences between drug and nondrug samples were nonsignificant and these were combined in subsequent analyses. Figure 1 provides an overview of reactivity in normal controls, and in the combined remitted and regressed samples.

Regressed schizophrenics revealed markedly impaired reactivity even on the initial presentation when novelty is maximal. Almost half failed to respond at all on trial 1: those who did showed a smaller mean OR magnitude than either remitted schizophrenic or control samples (Table 1). In both OR frequency and amplitude on trial 1 , the regressed sample was significantly below both controls (frequency, $p<.001$; amplitude, $p<.005)$, and remitted schizophrenics $(p<.001$; $\mathrm{p}<.05)$. However, as seen in Table 1 , deteriorated patients showed greater incidence of OR on trial 1 to the $25 \mathrm{ft}$ candle light than to the $5 \mathrm{ft}$-candle light $(\mathrm{p}<.02)$. In fact, initial OR frequency to the brighter light was

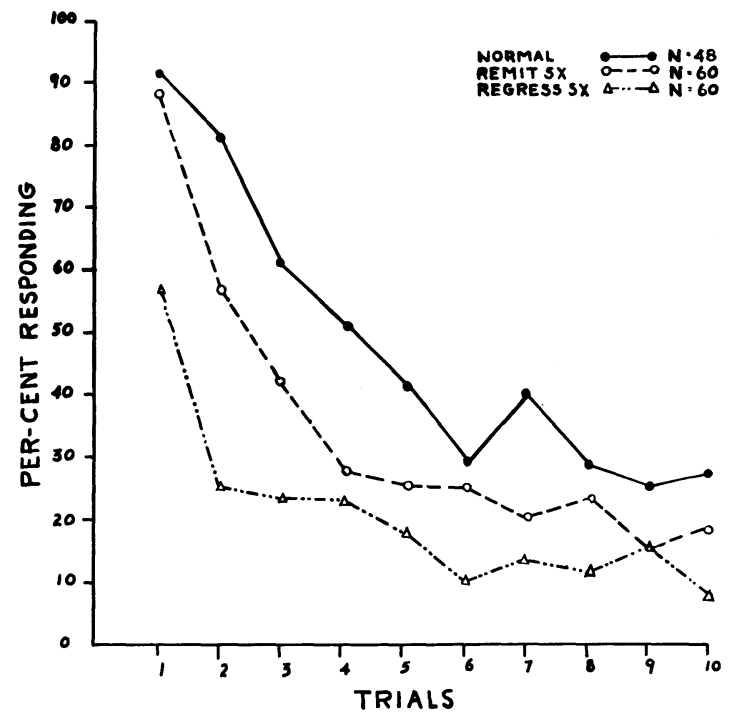

Fig. 1. Percentage of samples yielding OR response on each successive trial among normal controls, and remitted and regressed schizophrenics. 
ent from either controls $(p=.07)$, or remitted patients $(p<.50)$; regressed schizophrenics responded less frequently only when the $5 \mathrm{ft}$ candle stimulus was used (compared with either remitted patients or controls, $\mathrm{p}<.01$ ). Despite increased initial OR frequency when the brighter stimulus was used, neither mean amplitude nor overall oR frequency were affected.

The interaction with intensity was specific to regressed samples; stimulus intensity did not exhibit any other significant influence on OR function.

Remitted schizophrenics performed more like the control Ss in all OR dimensions except habituation. While all samples showed habituation (Fig. 1) (defined as number of trials until first appearance of two consecutive non-responses), remitted and regressed patients alike $(p>.30)$ habituated faster than did control Ss $(p<.001$ for each (Table 1$)$ ). This was the only instance in which all schizophrenics exhibited no differences among themselves, showing instead a common difference from controls. When a more conservative criterion was used, number of trials until three consecutive nonresponses, identical results emerged.

The reliability of these findings was underscored when retesting at approximately a two-month interval yielded virtually identical results in all comparisons.

Regressed samples had been hospitalized longer than remitted ones. However, hospitalization correlated significantly with only one OR function: a small negative relationship was indicated with total number of OR $(\mathrm{r}=-.29 ; \mathrm{p}=.01)$. The lack of further relationships indicates that the substantial differences between remitted and regressed schizophrenics are not simply functions of hospitalization. Even the difference in total number of OR's is not primarily a function of hospitalization: with this variable controlled through separate comparisons between remitted and regressed patients below the combined median hospitalization, and between those above it, remitted patients still revealed significantly greater overall OR activity ( $p=.03$ in each comparison).

\section{Discussion}

Results indicate that substantial OR impairment is associated primarily with manifest regression and disorganization within schizophrenia rather than with the fact of psychosis per se. Data from another study (using many of the present Ss) reveals that such impairment is not simply a by-product of general autonomic hypo-activity. Comparisons of autonomic arousal using electrodermal measurement did not indicate hypoarousal in chronic schizophrenics relative to controls. Thus, the decreased OR function described suggests impairment specifically in perceptual response among regressed schizophrenics, despite an apparently intact capacity for such arousal. This interpretation is supported by the increased initial OR frequency to the more intense stimulus and is consistent with theories describing schizophrenic disorganization as purposive, maintained to defend against the anxieties of external contact (e. g., Arieti, 1955). Increasingly intrusive (i. e., intense) stimuli may show increasing penetration of this barrier.

Faster habituation among schizophrenics conflicts with Russian reports and Pavlovian theory which sees in schizophrenia a state of cortical "protective inhibition" impairing control of sub-cortical centers. Since habituation is described as the result of cortically-mediated blocking of impulses to the reticular formation, faster habituation among schizophrenics is not in agreement with Pavlovian theory. Such faster habituation might be accomplished if schizophrenics categorize (identify) sensory-input on the basis of fewer, possibly more holistic determinants. Schizophrenia would thus be characterized generally by less detailed, less differentiated, more holistic perception.

It is unclear why increased hospitalization should be associated with reduced OR frequency while amplitude and habituation remain largely unaffected. Perhaps the influence of hospitalization is minimized because the bulk of the present patient samples has been long institutionalized ( $79 \%$ have been hospitalized over five years): the first four to five years may be more significant.

\section{References}

ARIETI, S. Interpretation of Schizophrenia, New York: Brunner, 1955.

LYNN, R. Russian theory and research on schizophrenia. Psychol. Bull., 1963, 60, 486-498.

SOKOLOV, E. N. Perception and the Conditioned Reflex, New York: Macmillian, 1963. SOKOLOV, E. N., PARAMONOVA, N. P., \& LOMONOSOV, M. V. Extinction of the orienting reflex. Pavlov J. Higher Nerv. Activ., 1961, 11, 1-8.

\section{Noter}

1. The writer thanks Jacob Cohen and Marvin Stein for their statistical advice and critical comments; John Bonsal for helping collect data and conduct statistical analyses; Anne Mattmann for preparing manuscripts, and William Royall for drawing the figure. 